\title{
DISCUSSION AFTER SESSION D
}

Fricke: How can one improve the accuracy of positions referred to reference stars from the measurements of the Palomar Survey?

Murray: In answer to Prof. Fricke I would say that more plates should be taken and measured in the Zone Surveys. The resolution of AGK3 is currently about 0.3 in each co-ordinate per star.

Stoy: The \pm 0 ". 3 quoted by Dr Hunstead probably reflects merely the errors in the Yale places of the reference stars, epoch 1933 brought forward to 1955 for the Sky Survey.

Improvement of this demands more photographic astrometric work.

Hunstead: Both the formal calculations and the results from repeat measurements suggest that it is the setting error on faint objects that dominates, not the catalogue positions.

Lacroute: I agree on the improvement of reference stars to improve the solutions. But if my trial is successful, that will be a contribution to the improvement of the solution by increasing the effective number of the reference stars. 